\title{
The sustained post-outburst brightness of Nova Per 2018, the evolved companion, and the long orbital period ${ }^{\star}$
}

\author{
U. Munari ${ }^{1}$, S. Moretti ${ }^{2}$, and A. Maitan ${ }^{2}$ \\ 1 National Institute of Astrophysics (INAF), Astronomical Observatory of Padova, 36012 Asiago (VI), Italy \\ e-mail: ulisse.munari@inaf.it \\ 2 ANS Collaboration, c/o Astronomical Observatory, 36012 Asiago (VI), Italy
}

Received 12 May 2020 / Accepted 21 June 2020

\begin{abstract}
Nova Per 2018 (= V392 Per) halted the decline from maximum when it was 2 mag brighter than quiescence and since 2019 has been stable at such a plateau. The ejecta have already fully diluted into the interstellar space. We obtained BVRIgrizY photometry and optical spectroscopy of V392 Per during the plateau phase and compared it with equivalent data gathered prior to the nova outburst. We find the companion star (CS) to be a G9 IV/III and the orbital period to be 3.4118 days, making V392 Per the longest known period for a classical nova. The location of V392 Per on the theoretical isochrones is intermediate between that of classical novae and novae erupting within symbiotic binaries, in a sense bridging the gap. The reddening is derived to be $E_{B-V}=0.72$ and the fitting to isochrones returns a $3.6 \mathrm{Gyr}$ age for the system and $1.35 M_{\odot}, 5.3 R_{\odot}$, and $15 L_{\odot}$ for the companion. The huge Ne overabundance in the ejecta and the very fast decline from nova maximum both point to a massive white dwarf (WD) $\left(M_{\mathrm{WD}} \geq 1.1-1.2 M_{\odot}\right)$. The system is viewed close to pole-on conditions and the current plateau phase is caused by irradiation of the CS by the WD still burning at the surface.
\end{abstract}

Key words. novae, cataclysmic variables

\section{Introduction}

Prior to its 2018 nova outburst (discovered by Y. Nakamura on 2018 Apr. 29.474 UT; cf. CBET 4515), V392 Per was a poorly studied cataclysmic variable (Bruch et al. 1987), eliciting little interest for its rare dwarf nova outbursts (only a few of which have been recorded) and faintness (mag 17.5 on blue photographic plates during quiescence). For some time it was considered lost because of the uncertainty of its position (Bruch et al. 1987) but was properly identified in later catalogs of cataclysmic variables (Downes et al. 1997). Early attempts to record its spectrum were scrubbed by the faintness of the system (Zwitter \& Munari 1994). The only published spectrum of V392 Per prior to its 2018 nova outburst was presented by Liu \& Hu (2000), who observed the star on 27 Nov 1998, when they estimated it was at $V=17.4$ mag.

V392 Per attracted wide interest during its recent 2018 nova outburst, in spite of the quickly approaching conjunction with the Sun. Spectral confirmation as a FeII-type nova was soon provided by Wagner et al. (2018), who measured $F W H M=5200 \mathrm{~km} \mathrm{~s}^{-1}$ and $E . W .=115 \AA$ for the emission, and $-2680 \mathrm{~km} \mathrm{~s}^{-1}$ and E.W. $=16 \AA$ for the absorption of the P-Cyg profile affecting $\mathrm{H} \alpha$. Within one day of the outburst announcement, V392 Per was detected as a strong gamma-ray source by Fermi-LAT (Li et al. 2018). Spectra on the following days (Tomov et al. 2018; Darnley et al. 2018a) supported a widening of the emission profiles $\left(F W H M=5600 \mathrm{~km} \mathrm{~s}^{-1}\right)$ and an

* Table 1 is only available at the CDS via anonymous ftp to cdsarc.u-strasbg.fr (130.79.128.5) or via http://cdsarc. u-strasbg.fr/viz-bin/cat/J/A+A/639/L10 even larger velocity for the P-Cyg absorption $\left(-4000 \mathrm{~km} \mathrm{~s}^{-1}\right)$. The main novelty was, however, the splitting of the emission component into three well separated peaks $(-2000,-250$, and $+1900 \mathrm{~km} \mathrm{~s}^{-1}$ ), which is a structure destined to last for the rest of the outburst (Darnley 2018a,b; Munari \& Ochner 2018; Munari et al. 2020; Stoyanov et al. 2020), and a close match to what observed and modeled by Munari et al. (2011) in the He/N-type nova V2672 Nova Oph 2009, seen very close to pole-on conditions.

The huge strength they recorded for neon emission lines led Munari \& Ochner (2018) to classify V392 Per as a Neon nova, which usually implies a massive progenitor for the current white dwarf (WD). Recently, Munari et al. (2020) found that V392 Per did not return to quiescence brightness, but instead remained stuck at $\sim 2$ mag above it, reminiscent of novae burning nuclearly at the surface for years after the end of the outburst (e.g., V723 Cas, Ochner et al. 2015). We will refer to such "sustained post-outburst brightness" of V392 Per as SPOB in the rest of this paper.

Radio observations during the first two weeks of the outburst were carried out with VLA and AMI-LA by Linford et al. (2018); the observations clearly detected the nova at 5.0, 7.0, and $15.5 \mathrm{GHz}$, and the spectral index supported a synchrotron emission mechanism. Finally, it was only following the solar conjunction that the Swift X-ray/UV satellite could be aimed at V392 Per. Darnley et al. (2018b) reported on these observations and on the uncertainty in modeling them given the low recorded flux level.

No information is currently available concerning the orbital period of V392 Per, and therefore the evolutionary status of 


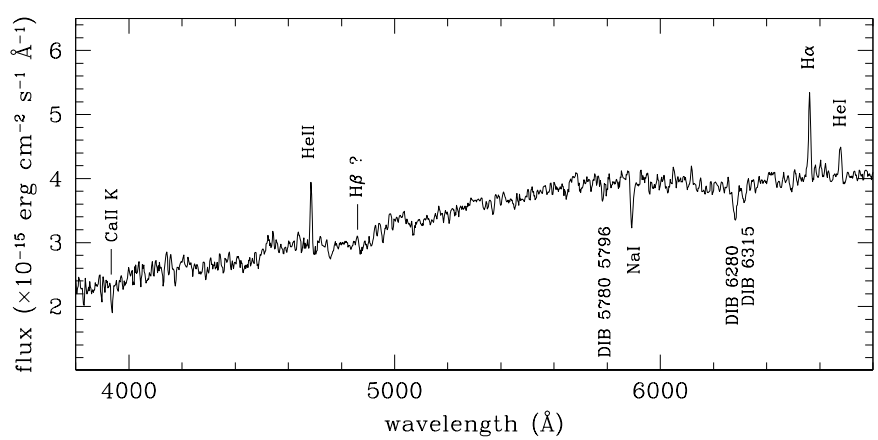

Fig. 1. Post-outburst spectrum of V392 Per for 2020 Apr 22.833 UT.

the donor star that Darnley \& Starrfield (2018) suggest it could be evolved. In this paper we compare and analyze the spectral energy distribution (SED) of V392 Per in quiescence and during SPOB, derive the physical properties of the donor star, and compare these properties with the orbital period of the system we determined.

\section{Observations}

A low resolution spectrum of V392 Per (range 3500-8000 , dispersion 2.31 $\AA \mathrm{pix}^{-1}$ ) was obtained on 2020 Apr 22.833 UT with the $\mathrm{B} \& \mathrm{C}$ spectrograph $+300 \mathrm{ln} \mathrm{mm^{-1 }}$ grating mounted on the Asiago $1.22 \mathrm{~m}$ telescope. It is presented in Fig. 1. It updates the spectrum for 2019 Dec 14 described in Munari et al. (2020), which was obtained with an identical instrument set-up.

Time-resolved optical photometry of V392 Per was obtained in the VRI bands using the ANS Collaboration $0.42 \mathrm{~m} \mathrm{f} / 5 \mathrm{New}-$ tonian telescope located in Bastia (Ravenna, Italy; ANS identifer ID 1205), for the purpose of detecting orbital modulation; V392 Per was observed for a total of 17 nights and 135 visits, that is to say about eight $V R I$ set per night on average. The presence of a field star of similar magnitude just 8 arcsec to the north (hereafter FS8) meant the photometry had to be carried out in a PSF-fitting mode. The results are given in Table 1. The quoted error is the quadratic sum of the Poissonian error on the variable and the error in the transformation from the instantaneous local photometric system to the Landolt equatorial standard system. Additional single-visit photometry, aimed to map the SED during SPOB, was obtained in Landolt BVRI and PanSTARRS grizY bands with ANS Collaboration ID 310 and Asiago 67/92 cm Schmidt telescopes; it is summarized in Table 2.

\section{Sustained post-outburst brightness (SPOB)}

As first noted by Munari et al. (2020), in 2019 V392 Per stopped declining in brightness toward its quiescence value. This SPOB plateau can be clearly seen in Fig. 2, which shows the $g$-band lightcurve of V392 Per reconstructed from observations collected by surveys monitoring the sky for transients ASAS-SN (Shappee et al. 2014; Kochanek et al. 2017) and ZTF (Bellm et al. 2019; Masci 2019). The large difference between the two sets of data is due to the large pixel-size of ASAS-SN (7.8 arcsec) that prevents separating V392 Per from FS8. In Table 2 we compare pre-outburst photometry of V392 Per (and FS8) from PanSTARRS PS1 (Chambers et al. 2016) with ours for two SPOB epochs in March 2020. While FS8 has remained the same, V392 Per is stuck at 2.0 mag brighter than quiescence in $g$, and this amount declines smoothly with wavelength down
Table 1. Time-resolved VRI photometry of V392 Per.

\begin{tabular}{ccccccc}
\hline \hline HJD & $V$ & Err. & $R$ & Err. & $I$ & Err. \\
\hline 871.246 & 15.018 & 0.008 & 14.346 & 0.009 & 13.618 & 0.009 \\
871.264 & 15.008 & 0.008 & 14.337 & 0.009 & 13.605 & 0.009 \\
871.282 & 15.054 & 0.008 & 14.381 & 0.009 & 13.667 & 0.010 \\
871.300 & 15.062 & 0.008 & 14.368 & 0.009 & 13.627 & 0.010 \\
871.321 & 15.081 & 0.009 & 14.396 & 0.009 & 13.691 & 0.010 \\
871.338 & 15.105 & 0.010 & 14.355 & 0.009 & 13.663 & 0.010 \\
871.356 & 15.059 & 0.009 & 14.377 & 0.009 & 13.676 & 0.010 \\
871.373 & 15.105 & 0.010 & 14.386 & 0.010 & 13.681 & 0.011 \\
871.391 & 15.118 & 0.010 & 14.417 & 0.010 & 13.706 & 0.010 \\
871.409 & 15.085 & 0.010 & 14.429 & 0.010 & 13.707 & 0.011 \\
871.427 & 15.037 & 0.010 & 14.364 & 0.010 & 13.653 & 0.011 \\
\hline
\end{tabular}

Notes. The complete table is only available in electronic form via CDS; a portion is shown here to provide guidance on its content. HJD is the heliocentric JD - 2458000 .

Table 2. Comparison between photometry prior to the nova outburst (epoch 2012.838 from PanSTARRS PS1) and during the SPOB phase (2020.194 and 2020.208), used to build the SEDs of Fig. 3.

\begin{tabular}{|c|c|c|c|c|c|c|c|c|c|}
\hline B & $g$ & V & $r$ & $R$ & $i$ & $I$ & $z$ & $Y$ & epoch \\
\hline \multicolumn{10}{|c|}{ V392 Per } \\
\hline \multirow{3}{*}{16.13} & 17.49 & & 16.37 & & 15.74 & & 15.38 & 15.09 & 2012.838 \\
\hline & 15.53 & 15.10 & 14.76 & 14.46 & 14.28 & 13.73 & & & 2020.194 \\
\hline & & 14.99 & 14.64 & & 14.17 & 13.66 & 13.89 & 13.75 & 2020.208 \\
\hline \multicolumn{10}{|c|}{ Field star $8^{\prime \prime}$ to the north (FS8) } \\
\hline \multirow{3}{*}{16.06} & 15.43 & & 14.79 & & 14.50 & & 14.35 & 14.24 & 2012.838 \\
\hline & 15.46 & 15.08 & 14.82 & 14.57 & 14.53 & 14.03 & & & 2020.194 \\
\hline & & 15.09 & 14.81 & & 14.53 & 14.04 & 14.38 & 14.29 & 2020.208 \\
\hline
\end{tabular}

to $1.3 \mathrm{mag}$ at $Y$. This indicates that the source responsible for the SPOB is hotter than V392 Per was, as a whole, in quiescence.

The higher temperature conditions of V392 Per during SPOB are confirmed by the spectrum in Fig. 1 when compared with the quiescence spectrum presented by Liu \& Hu (2000). The latter was characterized by $\mathrm{H} \alpha / \mathrm{H} \beta \sim 1$ and HeII $4686 / \mathrm{H} \beta \sim 0.5$. In the spectrum of Fig. $1, \mathrm{H} \beta$ is possibly too weak to be seen while HeII 4686 is very strong, rivaling $\mathrm{H} \alpha$ in intensity; HeI 6678 also stands out clearly. It is also worth noting that the only remaining signature of the nova ejecta, [OIII] 5007, has further declined compared to its appearance in the spectrum for 2019 Dec 14, described by Munari et al. (2020), and is now just barely visible, marking the final end of the nova phase.

\section{Reddening}

Reddening plays a crucial role in deriving the evolutionary status of the companion star (CS) to the WD in V392 Per. There are only two published estimates, which are in gross disagreement.

The Bayestar2019 3D map of Galactic extinction by Green et al. (2019) reports $E_{B-V}=0.63_{-0.03}^{+0.02}$ at the Gaia DR2 distance $(3.88 \mathrm{kpc})$ along the general line of sight to V392 Per. Tomov et al. (2018), on their high resolution $\left(R_{P}=30000\right)$ spectra of V392 Per, found the diffuse interstellar bands at 5780, 5797, and $6614 \AA$, as well as the interstellar lines of $\mathrm{NaI}(5890,5896 \AA)$ and $\mathrm{KI}(7665,7699 \AA)$ to be very strong; they derived $E_{B-V}=$ $1.18 \pm 0.10$ from their equivalent widths.

The high $E_{B-V}$ estimated by Tomov et al. (2018) is in contrast to the observed photometric color. van den Bergh \& Younger (1987) measured a mean intrinsic color $(B-V)$ 。 $=$ 


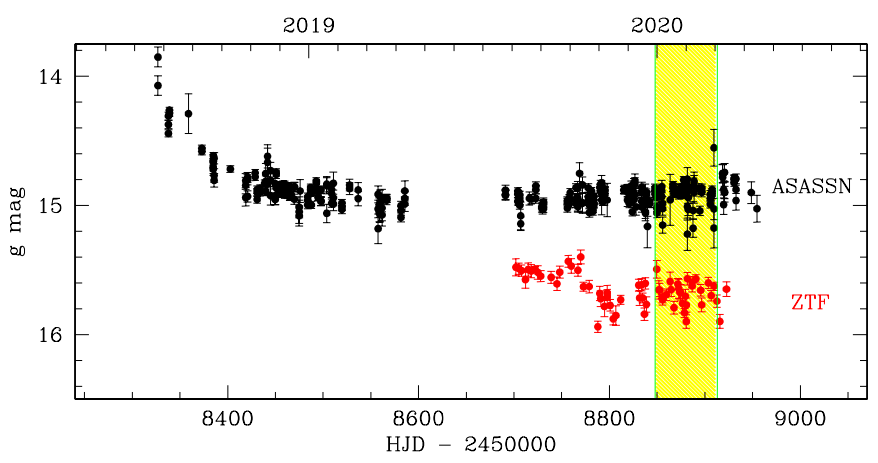

Fig. 2. Post-outburst $g$-band lightcurve of V392 Per from ASAS-SN and ZTF data. The region in yellow is the interval covered by our timeresolved observations in search of the orbital period.

$+0.23 \pm 0.06$ for novae at optical maximum, and $(B-V)_{\circ}=$ $-0.02 \pm 0.04$ at $t_{2}$. The AAVSO lightcurve shows that V392 Per was slightly past maximum when, on 2018 Apr. 30.116 UT, $B-V=0.92$ was measured by S. Kiyota (cf. CBET 4515, where no estimate of the error is provided), from which we derive $E_{B-V}=0.72 \pm 0.06$.

\section{Spectral energy distribution (SED)}

V392 Per in quiescence is present in several all-sky photometric catalogs, but in most of them it is blended with FS8 and/or the different bands were observed at widely different epochs. The only two catalogs resolving V392 Per from FS8 and presenting simultaneous multi-band data are PanSTARRS PS1 grizY bands observed on 2012.194 (Chambers et al. 2016) and 2MASS $J H K_{S}$ bands observed on 1999.773 (Cutri et al. 2003). Their combined SED is presented in Fig. 3 (black line) and is clearly bi-modal, with the WD+accretion disk dominating over griz and the CS on $J H K_{S}$, with the $Y$ band marking the smooth transition between the two. This very fact indicates that the brightness of V392 Per was similar at the time of the PanSTARRS and 2MASS visits, and consequently their data can be safely combined into a unique SED, marked by the black solid line in Fig. 3. We deconvolved it into two components by fitting two second-order polynomials, represented by the blue and red lines in Fig. 3. We did not attempt to fit more complex distributions in view of the very few available points (eight) constraining the overall fit, and the uncertainty of any deviation from standard photospheric and disk models due to irradiation and viewing angle. The adopted simplification, however, has no practical consequence on the conclusions reached in this paper.

The original 2MASS photometry of V392 Per in quiescence reads $J=13.766( \pm 0.031), H=13.290( \pm 0.038)$, and $K_{s}=13.062( \pm 0.037)$. After subtracting the contribution of the hot component as in Fig. 3, they become (red line) $J \sim 14.099$, $H \sim 13.392$, and $K_{s} \sim 13.092$. It is relevant to note the very small amount of the subtraction affecting the $K_{s}$ band, which constrains the evolutionary status of the CS.

The SED of V392 Per during SPOB has been derived at two nearby epochs, 2020.194 and 2020.208, and plotted in Fig. 3 as green and blue rhombuses from the data listed in Table 2. The SED at these two epochs is the same except for an offset of $\Delta m \sim 0.1 \mathrm{mag}$, which corresponds to the typical amplitude of brightness variation during SPOB. A second-order polynomial fit is plotted as a cyan line. Subtracting the SED of the hot component in quiescence (blue line) from it results in the dashed black line in Fig. 3; this represents the source of the extra

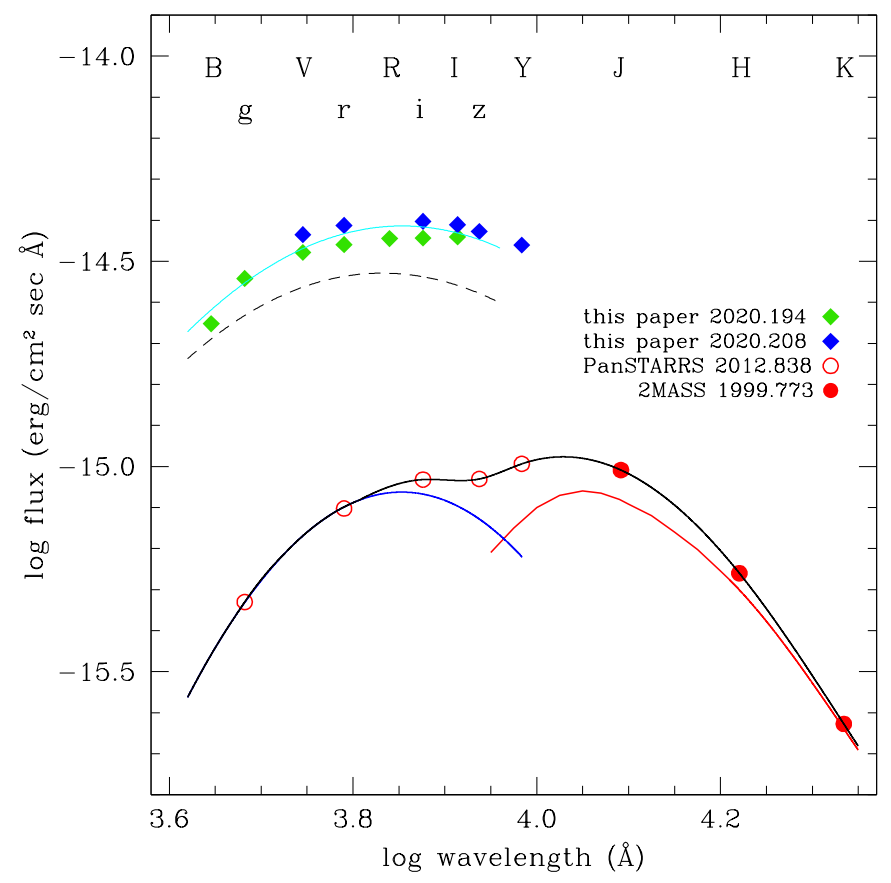

Fig. 3. Spectral energy distribution of V392 Per in quiescence (solid black line), split into the hot (blue line) and cool (red line) components, and during SPOB (cyan line). The dashed black line is the difference between the blue and cyan lines and represents the excess emission during the SPOB phase (see Sect. 5).

Table 3. Gross properties of the source responsible for extra brightness of SPOB (the dashed black line in Fig. 3) as a function of $E_{B-V}$ reddening.

\begin{tabular}{cccc}
\hline \hline$E_{B-V}$ & $\begin{array}{c}T_{\text {eff }} \\
\left({ }^{\circ} \mathrm{K}\right)\end{array}$ & $\begin{array}{c}L_{\text {opt }} \\
\left(L_{\odot}\right)\end{array}$ & $\begin{array}{c}r \\
\left(R_{\odot}\right)\end{array}$ \\
\hline 0.63 & 7500 & 42 & 3.85 \\
0.72 & 8750 & 55 & 3.25 \\
1.18 & 38000 & 329 & 0.38 \\
\hline
\end{tabular}

Notes. The $T_{\text {eff }}$ is the temperature of the fitting black-body and $L_{\text {opt }}$ its luminosity radiated within the optical window $(0.3-1.0 \mu \mathrm{m}) ; r$ is the radius of a black-body emitting the equivalent bolometric luminosity.

brightness of V392 Per during SPOB. We have fitted back-body distributions to the dashed line in Fig. 3 as a function of $E_{B-V}$ and the results are reported in Table 3: with increasing reddening, the temperature and luminosity go up and the dimensions decline.

\section{Isochrones fitting}

The 2MASS photometry of the CS in V392 Per (red line in Fig. 3) is compared with Padova isochrones (Bressan et al. 2012) in Fig. 4 for the values of $E_{B-V}$ listed in Sect. 4 and Table 3. We have also plotted the position of some reference objects: (a) two cataclysmic variables of the same UGEM type as V392 Per, one in quiescence and the other in outburst (U Gem with Period $=4.2$ and SS Cyg with $P=6.6 \mathrm{~h}$, respectively); (b) four novae that have some of the longest known orbital periods $(P=0.6042$ days for V841 Oph, $P=0.6905$ days for V368 Aql, $P=1.2306$ days for U Sco, and $P=1.9968$ days for GK Per); and (c) two recurrent novae with giant secondaries (T CrB with $P=227$ days and RS Oph with $P=454$ days). The results of fitting 


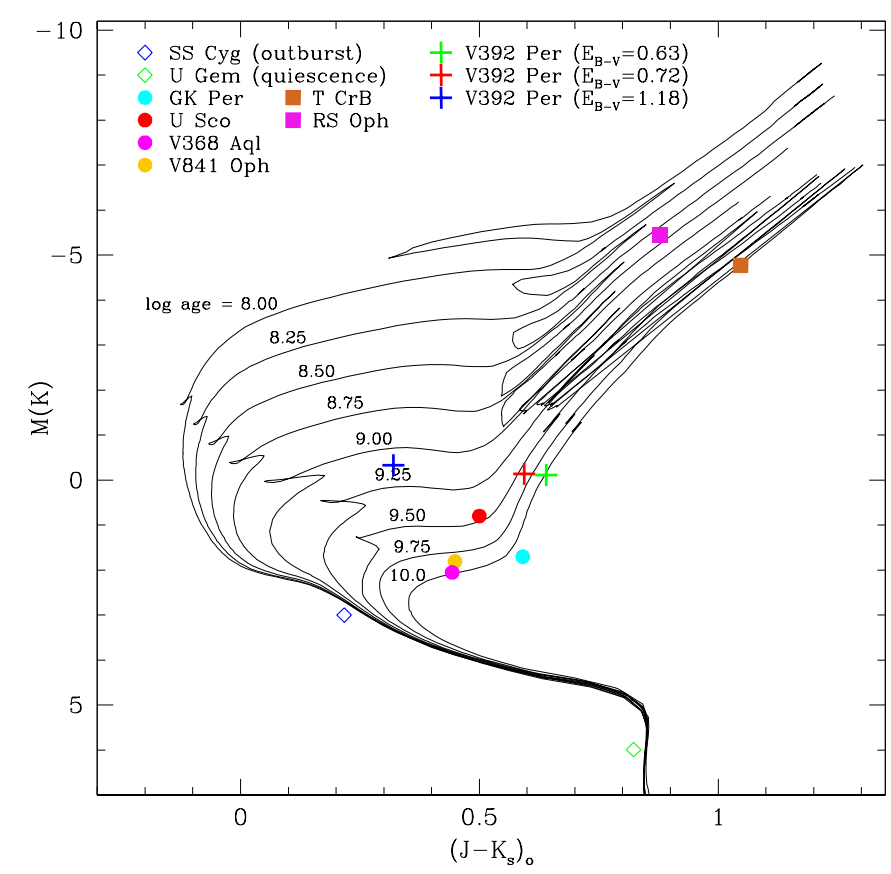

Fig. 4. Position of the CS in V392 Per (red line in Fig. 3) on the Padova isochrones for the 2MASS plane, dereddened for the $E_{B-V}$ values listed in Sect. 4 and Table 4. Dereddened 2MASS photometry of some representative novae and cataclysmic variables with accurate Gaia parallax is plotted for comparison (see text for details).

Table 4. Properties of the CS to the WD in V392 Per from fitting to Padova isochrones on the 2MASS plane, as a function of $E_{B-V}$ reddening.

\begin{tabular}{ccccccccc}
\hline \hline$E_{B-V}$ & $\begin{array}{c}\text { Age } \\
(\mathrm{Gyr})\end{array}$ & $\begin{array}{c}M_{\mathrm{CS}} \\
\left(M_{\odot}\right)\end{array}$ & $\begin{array}{c}T_{\mathrm{CS}}^{\mathrm{eff}} \\
(\mathrm{K})\end{array}$ & $\begin{array}{c}L_{\mathrm{CS}} \\
\left(L_{\odot}\right)\end{array}$ & $\begin{array}{c}R_{\mathrm{CS}} \\
\left(R_{\odot}\right)\end{array}$ & $\begin{array}{c}M_{\mathrm{WD}} \\
\left(M_{\odot}\right)\end{array}$ & $\begin{array}{c}a \\
\left(R_{\odot}\right)\end{array}$ & $\begin{array}{c}P \\
(\mathrm{days})\end{array}$ \\
\hline 0.63 & 10.0 & 1.03 & 4740 & 13 & 5.36 & 0.58 & 12.58 & 4.07 \\
& & & & & & 1.10 & 14.49 & 4.38 \\
0.72 & 3.6 & 1.35 & 4875 & 15 & 5.34 & 0.61 & 11.81 & 3.36 \\
& & & & & & 1.10 & 13.37 & 3.61 \\
1.18 & 1.4 & 1.92 & 5915 & 31 & 5.31 & 0.66 & 11.20 & 2.70 \\
& & & & & & 1.10 & 12.39 & 2.90 \\
\hline
\end{tabular}

Notes. The $J H K_{S}$ photometry of V392 Per is corrected for the contribution in quiescence of the hot component (cf. red line in Fig. 3). The orbital separation $(a)$ and period $(P)$ are computed assuming that CS fills its Roche lobe (see Sect. 6 for details).

to isochrones (for solar metallicity) is summarized in the first six columns of Table 4. A higher reddening corresponds to a younger system and a more massive, more luminous, and hotter CS.

The remaining columns of Table 4 are devoted to deriving the orbital period expected under the assumption that the CS (of radius $R_{\mathrm{CS}}$ ) fills its Roche lobe, as it is customary to assume in cataclysmic variables and novae (cf. Warner 1995). To this aim, two alternatives are considered for the mass of the WD. The first is the expected WD mass (from Cummings et al. 2000) produced by a progenitor, which on the main sequence had a mass $10 \%$ larger than the current CS. The second alternative is a WD mass of $1.10 M_{\odot}$, a minimum (Doherty et al. 2015) for a WD of ONeMg composition taking into consideration the neon overabundance in the ejecta of V392 Per reported by Munari \&

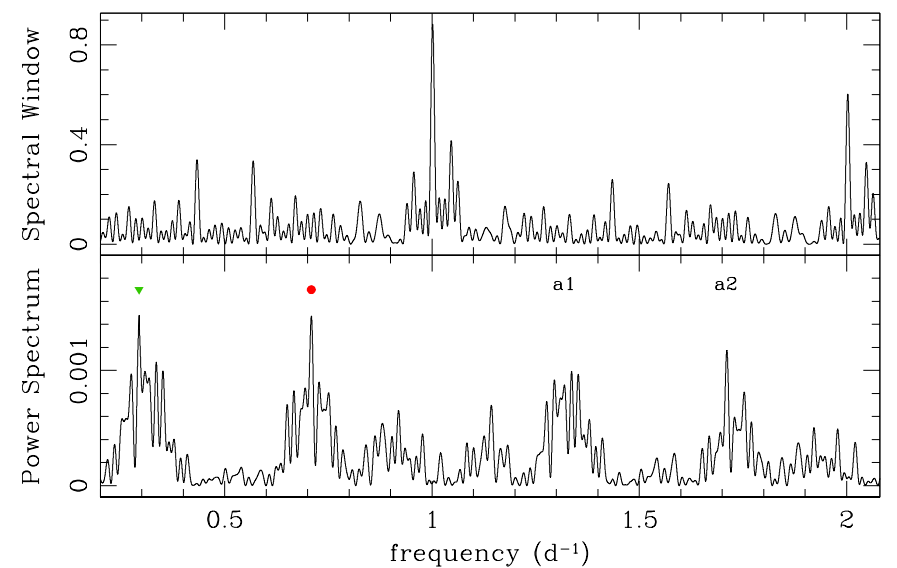

Fig. 5. Spectral window and power spectrum over the 5.0-0.5 day interval for the Fourier analysis of $V$-band data in Table 1 . The green triangle and red dot mark the 3.4118 and 1.4118 day periods, respectively, and $a 1$ and $a 2$ mark their respective one-day aliases.

Ochner (2018). A high mass for the WD $\left(M \geq 1.2 M_{\odot}\right)$ is also supported by the very short decline times, $t_{2}=3$ and $t_{3}=11$ days, measured by Stoyanov et al. (2020), relative to Kato \& Hachisu (1994) theoretical models.

The requirement for the CS to fill its Roche lobe sets the relation between stellar radius and orbital separation. If $q=$ $M_{\mathrm{CS}} / M_{\mathrm{WD}}$ is the mass ratio, the orbital separation follows from the Eggleton (1983) relation:

$\frac{R_{\mathrm{CS}}}{a}=\frac{0.49 q^{2 / 3}}{0.6 q^{2 / 3}+\ln \left(1+q^{1 / 3}\right)}$.

The orbital period in the last column of Table 4 is then computed from Kepler's third law: $\left(M_{\mathrm{CS}}+M_{\mathrm{WD}}\right) P^{2}=a^{3}$.

The main results of Fig. 4 and Table 4 are that the evolutionary status of the CS requires the orbital period of V392 Per to be longer than those of classical novae. The knowledge of the orbital period (see next section) considerably narrows the uncertainty on properties of the CS left open by the \pm 0.06 mag error on the adopted $E_{B-V}=0.72$ reddening.

\section{The orbital period}

Our time-resolved VRI observations in Table 1 have been examined for periodicities. For the analysis we adopted Fourier transforms following the formulation by Deeming (1975), and checked the results with the phase dispersion minimization algorithm of Stellingwerf (1978). The Fourier power spectrum and spectral window over the 5.0-0.5 day interval are plotted in Fig. 5; the results outside this interval are insignificant.

There are two clear peaks in the power spectrum, at 3.4118 and 1.4107 days, as well as at their one-day aliases (the oneday aliases are marked respectively with $a 1$ and $a 2$ in Fig. 5). Their broad pedestals are caused by the $\sim 70$ day duration of the observing campaign and the grouping of observations to avoid bright Moon phases. It is worth noting that the two peaks relate as $1 / 3.4118+1 / 1.4107=1.00$ days, so they are not mutually independent.

Determining which is the true period and which is the alias can be done with the help of Table 4 . The 3.4118 day period corresponds to highly reasonable values for the age and mass of the $\mathrm{CS}$, and match the reddening $\left(E_{B-V}=0.72\right)$ derived from the photometric color of the nova at maximum. On the contrary, a period of 1.4107 days would require an impossibly high 


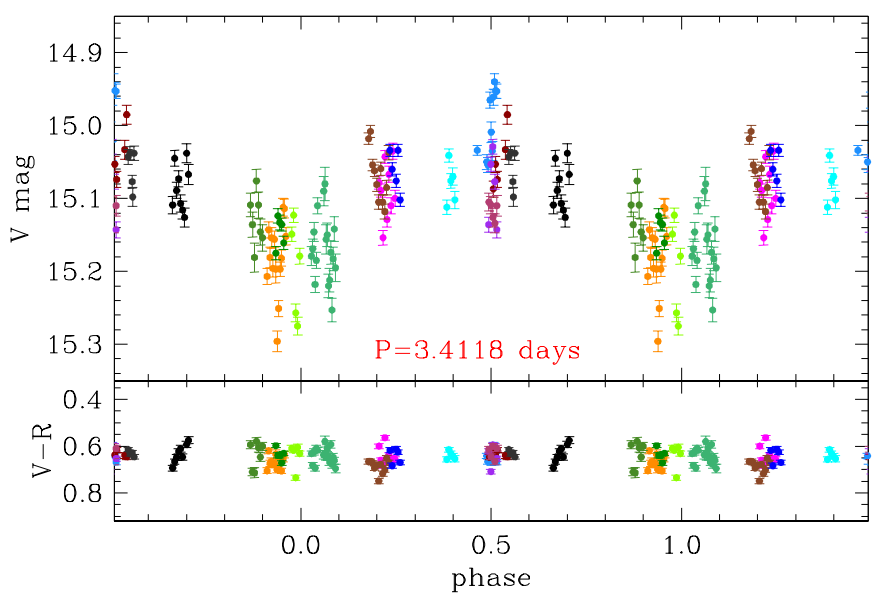

Fig. 6. Phased light-curves for the $V$-band data in Table 1 according to ephemeris in Eq. (2). Different colors mark data from different nights. The bars are the total error budget listed in Table 1.

value for $E_{B-V}(\geq 1.8 \mathrm{mag})$ and a very young age for the system $(\leq 0.3 \mathrm{Gyr})$, much younger than is typical for cataclysmic variables and novae in general. The robustness of the 3.4118 day period is also confirmed by the pre-whitening analysis of the data (see Appendix A). The photometry of V392 Per in Table 1 is phase-plotted in Fig. 6 following the ephemeris:

$\min (\mathrm{V})=2458853.58( \pm 0.07)+3.4118( \pm 0.0013) \times E$.

\section{System properties}

From 2MASS photometry, orbital period, and the assumption of Roche-lobe filling, it follows that the CS in V392 Per is an early giant that has just begun the ascent along the giant branch, and it is characterized by $5.34 R_{\odot}, 1.35 M_{\odot}, 15 L_{\odot}$, and $T_{\text {eff }}=4875 \mathrm{~K}$. Such values correspond to a G9 IV-III star (estimated from parameters listed in Cox 2000). Such a binary system is highly unusual among classical novae, and appears to bridge the gap with novae erupting within symbiotic stars, as its location in Fig. 4 clearly shows. The presence of an early giant in the system is in agreement with the very early detection of $\gamma$-rays and synchrotron radio emission within one day of nova discovery, probably caused by fast ejecta slamming onto a pre-existing and slow circumstellar medium formed by the CS wind (cf. Abdo et al. 2010). None of the preliminary descriptions currently available report evidence from spectra of V392 Per of a deceleration of the ejecta or narrow components from flash-ionized wind from the companion; however, such deficiencies have already been seen in the nova outbursts of V3890 Sgr (Munari \& Walter 2019) and Nova Sco 2014 (Munari \& Banerjee 2018), which harbor much cooler and more luminous giants with, presumably, much larger mass-loss rates into the circumstellar space than V392 Per.

The brightness of V392 Per at the peak of nova outburst is $V \sim 6.3$ and the mean in quiescence is $V \sim 16.9$ (from AAVSO lightcurve). The limited $\Delta V \sim 10.6$ mag outburst amplitude falls much shorter than the $13.5 \mathrm{mag}$ value implied by the amplitude versus $t_{2}$ decline-time relation for face-on systems (Fig. 5.4 in Warner 1995, updated to the zero-point by Selvelli \& Gilmozzi 2019), with an obviously increasing discrepancy for larger orbital inclinations. The brighter-than-usual magnitude in quiescence is further evidence of the presence of a CS in V392 Per that is much brighter than in classical novae, whose optical brightnesses are completely dominated by the accretion disk. In this sense, it is worth noting that at the distance $\left(3.88_{-0.64}^{+0.98} \mathrm{kpc}\right.$, from Gaia DR2 parallax) and reddening $\left(E_{B-V}=0.72\right)$ affecting V392 Per, the G9 IV-III companion would shine at $V \sim 17.35$, adopting the absolute magnitude tabulated by Sowell et al. (2017). To add to $V \sim 16.9$ in quiescence, the WD+disk needs to emit at $V \sim 18.0$, brighter than the $V \sim 18.8$ implied by the MMRD relation (magnitude at maximum versus rate of decline) as calibrated on Gaia distances by Selvelli \& Gilmozzi (2019), and scaled to face-on orbital inclination. The wider orbital separation and higher mass transfer in V392 Per are able to support a disk that is larger and brighter than in classical novae.

If a low orbital inclination of V392 Per is favored by the above line of reasoning on the brightness in quiescence, it is actually required by the profile of emission lines observed during the 2018 nova outburst. The bulk velocity of the approaching and receding components of the bipolar flow (-2000 and $+1900 \mathrm{~km} \mathrm{~s}^{-1}$; Tomov et al. 2018) are among the largest ever observed in classical novae; such a wide split can only be obtained for a low orbital inclination and close to face-on orientation. At low orbital inclination, the side of the CS that is irradiated by the burning WD is in constant sight, and a limited deviation from $i=0^{\circ}$ is responsible for the small amplitude ( $\Delta m \sim 0.1 \mathrm{mag}$ ) of the orbital modulation (Fig. 6). The constancy of photometric color along the orbital motion (Fig. 6) agrees with the inferred low inclination. For comparison, the orbital modulation caused by irradiation in V723 Cas amounts to $\sim 2$ mag given the much larger orbital inclination of that system $\left(\sim 65^{\circ}\right.$, Ochner et al. 2015). The irradiated side of the CS as the source of the extra optical brightness of V392 Per during SPOB is confirmed by the large dimension derived in Table 3 for the $E_{B-V}=0.72$ case (implied by the $P=3.4118$ day orbital period and confirmed by the color at nova maximum). This scenario is also in agreement with the small equivalent width of the emission lines in the spectrum shown in Fig. 1: they need the high temperature of an irradiated disk to form but have to emerge over the rethermalization spectrum of the irradiated side of the CS (which is too cold to originate any of these emission lines, cf. Table 3).

Acknowledgements. We thank P. Selvelli (INAF Trieste) and R. Raddi (UC, Barcelona) for useful discussions and P. Ochner (Univ. of Padova), S. Dallaporta, A. Frigo, V. Andreoli, M. Graziani and S. Tomaselli (ANS Collaboration), for collecting some of the photometric data used in this project.

\section{References}

Abdo, A. A., Ackermann, M., Ajello, M., et al. 2010, Science, 329, 817 Bellm, E. C., Kulkarni, S. R., Graham, M. J., et al. 2019, PASP, 131, 018002 Bressan, A., Marigo, P., Girardi, L., et al. 2012, MNRAS, 427, 127 Bruch, A., Fischer, F.-J., \& Wilmsen, U. 1987, A\&AS, 70, 481 Chambers, K. C., Magnier, E. A., Metcalfe, N., et al. 2016, ArXiv e-prints [arXiv:1612.05560]

Cox, A. N. 2000, Astrophysical Quantities, 4th edn. (NewYork: Springer) Cummings, J. D., Kalirai, J. S., Tremblay, P. E., et al. 2000, ApJ, 866, 21 Cutri, R. M., Skrutskie, M. F., van Dyk, S., et al. 2003, VizieR Online Data Catalog: II $/ 246$

Darnley, M. J. 2018a, ATel., 11846

Darnley, M. J. 2018b, ATel., 11872

Darnley, M. J., \& Starrfield, S. 2018, RNAAS, 2, 24

Darnley, M. J., Copperwheat, C. M., Harvey, E. J., et al. 2018a, ATel., 11601

Darnley, M. J., Page, K. L., Beardmore, A. P., et al. 2018b, ATel., 11905

Deeming, T. J. 1975, Ap\&SS, 36, 137

Doherty, C. L., Gil-Pons, P., Siess, L., et al. 2015, MNRAS, 446, 2599

Downes, R., Webbink, R. F., \& Shara, M. M. 1997, PASP, 109, 345

Eggleton, P. P. 1983, ApJ, 268, 368

Green, G. M., Schlafly, E., Zucker, C., et al. 2019, ApJ, 887, 93 
Kato, M., \& Hachisu, I. 1994, ApJ, 437, 802

Kochanek, C. S., Shappee, B. J., Stanek, K. Z., et al. 2017, PASP, 129, 4502

Li, K.-L., Chomiuk, L., \& Strader, J. 2018, ATel., 11590

Linford, J. D., Bright, J., Chomiuk, L., et al. 2018, ATel., 11647

Liu, W., \& Hu, J. Y. 2000, ApJS, 128, 387

Masci, F. J., Laher, R. R., Rusholme, B., et al. 2019, PASP, 131, 018003

Munari, U., \& Banerjee, D. P. K. 2018, MNRAS, 475, 508

Munari, U., \& Ochner, P. 2018, ATel., 11926

Munari, U., \& Walter, F. M. 2019, ATel., 13099

Munari, U., Ribeiro, V. A. R. M., Bode, M. F., et al. 2011, MNRAS, 410, 525

Munari, U., Moretti, S., \& Maitan, A. 2020, ATel., 13381

Ochner, P., Moschini, F., Munari, U., et al. 2015, MNRAS, 454, 123

Selvelli, P., \& Gilmozzi, R. 2019, A\&A, 622, A186

Shappee, B. J., Prieto, J. L., Grupe, D., et al. 2014, ApJ, 788, 48

Sowell, J. R., Trippe, M., Caballero-Nieves, S. M., et al. 2017, AJ, 134, 1089

Stellingwerf, R. F. 1978, ApJ, 224, 953

Stoyanov, K. A., Tomov, T., Stateva, I., et al. 2020, Bulgarian AJ, 32, 63

Tomov, T., Stateva, I., Georgiev, S., et al. 2018, ATel., 11605

van den Bergh, S., \& Younger, P. F. 1987, A\&AS, 70, 125

Wagner, R. M., Terndrup, D., Darnley, M. J., et al. 2018, ATel., 11588

Warner, B. 1995, Cataclysmic Variable Stars (Cambridge Univ. Press)

Zwitter, T., \& Munari, U. 1994, A\&AS, 107, 503

\section{Appendix A: Fourier analysis of pre-whitened photometric data}

A Fourier analysis was carried out on the same input photometric data used for the periodogram of Fig. 5, after pre-whitening for the 3.4118 day period. The results are shown in Fig. A.1. The absence of residual peaks confirms the correctness of the derived period.

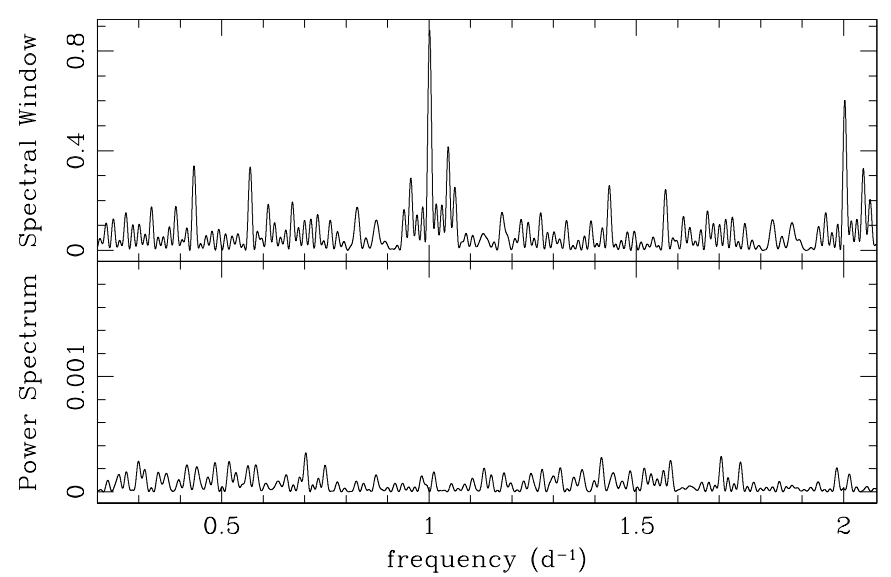

Fig. A.1. Spectral window and power spectrum over the 5.0-0.5 day interval for the Fourier analysis of $V$-band data in Table 1 after prewhitening for the 3.4118 day period. 